\title{
Implementation of Automatic Identification System for Safety Operation of Maritime Installation
}

\author{
Ketut Buda Artana ${ }^{1, a, *}$, Emmy Pratiwi', Dwi Kristianto ${ }^{2, b}$, and Dwi Suasti Antara ${ }^{2}$ \\ ${ }^{1}$ Department of Marine Engineering - PUI KEKAL, Institut Teknologi Sepuluh Nopember, \\ Surabaya, Indonesia \\ ${ }^{2}$ AISITS Surabaya - PUI KEKAL, Institut Teknologi Sepuluh Nopember, Surabaya Indonesia \\ a.ketutbuda@its.ac.id,b.aisits@its.ac.id \\ *corresponding author
}

Keywords: $\quad$ AISITS, Ship's Incident, Real Time, Early Monitoring System

Abstract: The increase in the proportion of the world's cargo transported by ships is increasing every year. This certainly encourages the growth of the number of vessels which ultimately can lead to increased risks, especially the risks to maritime installation due to ships operating around it. The most frequent risks are inter-ship collision, ship collision with offshore platform, or danger to subsea pipeline due to dropped anchor, dragged anchor and ship sinking. AISITS developed by ITS Surabaya is a real time-early warning system that allows us to maintain the safety of maritime installation against hazards generated by nearby vessels. This application can provide early warning before the ship accident occurs and can provide notification of a response that needs to be given to prevent incidents from happening. This application can also identify the danger score of the ship, the level of urgency inspection of the ship, vessel tracking that allows the reconstruction of accidents and other applications including ship service at the port. This application is expected to significantly reduce ship collision rates, particularly in relatively narrow territorial waters. AISITS also provides customized systems that allow the addition of various algorithms to support applicability while at the same time reducing the investment required for hardware and software.

\section{Introduction}

Accidents and incidents involving ships are not only fatal to ships, cargo, crew or maritime installations, yet they also have a direct impact on the marine environments. The cost incurred are often enormous, including costs incurred to deal with pollution, compensation for existing business processes, and loss of business opportunities due to incidents. Based on the official data of Indonesian Government in 2009, there were 293 reported major casualty case: ship sinking (31\%), ship grounding (25\%), ship collision (18.27\%), ship fires $(9.67 \%)$ and others $(16.06 \%)$. The cause of the accident was human error (78.45\%), technical error (9.67\%), weather condition (1.07\%), and 10.75\% due to combination of weather and technical error [1]. Based on IMO Resolution MSC.74 (69) [2], AIS (Automatic Identification System) has been forced to use in the vessel operations, for ship with capacity of over 300GT. Utilization of AIS device is intended to reduce the probability of ships collision. AIS system automatically sends dynamic data such as location, speed, course and heading as well as static data such as width, length, draft and other supplementary information. Today, AIS is 
not only used in ships, but furthermore, it also used at base station to monitor ship traffic which is VTS (Vessel Traffic Service) [3].

This paper presents the results of research conducted by the Laboratory Reliability and Safety ITS Surabaya embodied in an application called AISITS. This real time application contains several features such as early warning system (EWS) of ship collision hazard with maritime installation and subsea pipeline. EWS automatically sends a warning notification to all the port and ship stakeholders via email, big screen, or mobile notification (Fig). AISITS is also equipped with features to determine the level of the ship's danger (danger score) which shows the quantity of ship's luminance by considering the ship age, classification, ship type, ship flag, and other variables [4], [5]. The ship tracking feature also allows us to conduct forensic studies of ship collisions, including some additional features related to emissions, ship services and so on.

AISITS and its features is a compilation of academic studies conducted by ITS in cooperation with several other universities. This paper does not present the underlying algorithm of the features in the application, but rather shows how the application is used to support the operational safety of ships at sea. AISITS was developed through a series of code developments and has been through testing both in laboratories and in port testing (AIS Station Port of Tanjung Perak Surabaya). This application can also be accessed via web at www.aisits.id. The algorithm in details of existing features can be further asked and discussed to the author via email.

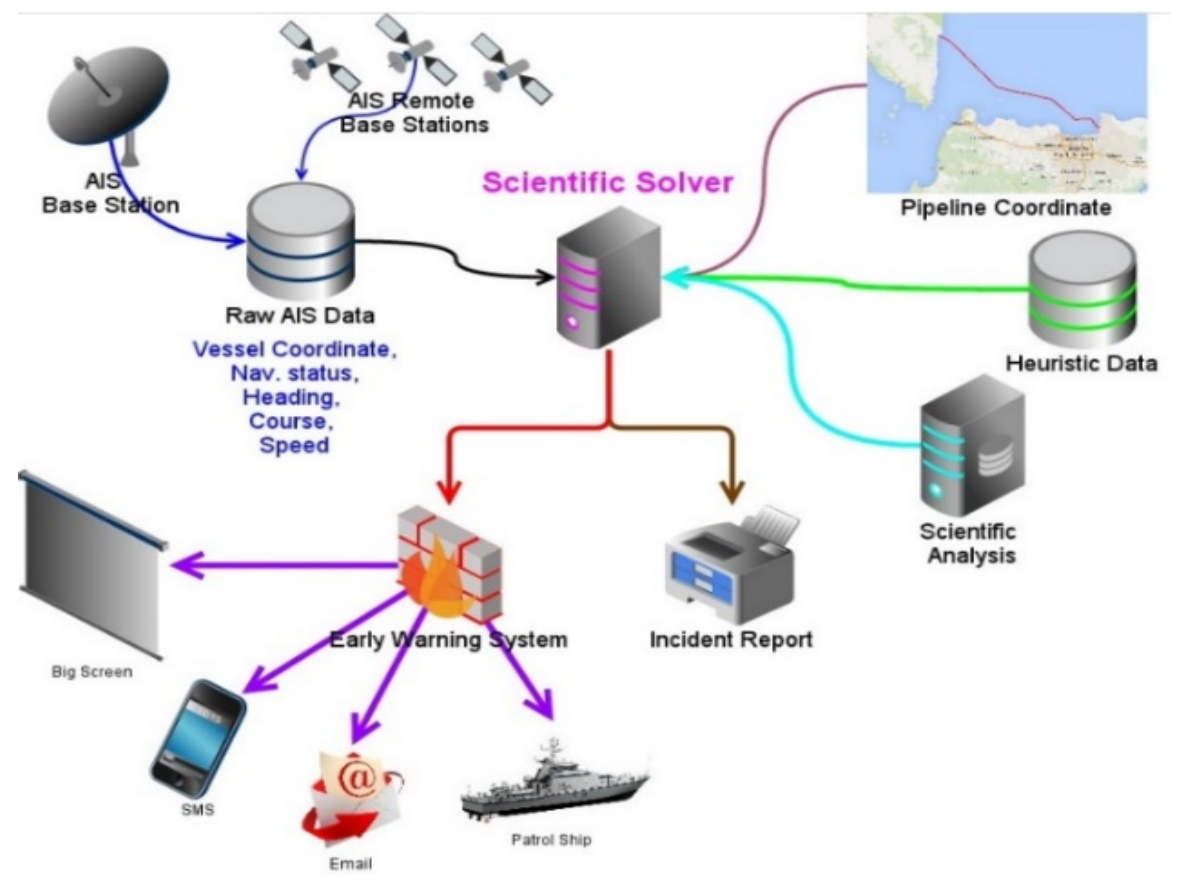

Figure 1: Main concept of AISITS.

\section{Automatic Identification System}

The Automatic Identification System (AIS) is an automatic tracking system used on ships and by Vessel Traffic Service (VTS) to identify and search vessels by electronically exchanging data with other nearby ships and AIS Base stations [6]. The AIS information complements marine radar, which continues to be the primary method of avoiding collisions for sea transport. Through the visualization system on board ship presents the bearing and distance of nearby ships in a radar-like display format, AIS functions can significantly improve the safety of ship operations[5]. 
AIS provides a graphical display on board where the ship's information provided by AIS equipment. The information of ship data obtained from AIS such as unique identification, position, course, and speed, can be displayed on the screen or ECDIS. AIS is supposed to assist a ship's watchstanding officers and allow maritime authorities to track and monitor the ship movements [5].

AIS is a technology based on Very High Frequency (VHF) radio communication which used to track and monitor vessel movements. AIS can be viewed in chart plotter or computer. AIS is a maritime safety and vessel traffic system that imposed by the International Maritime Organization (IMO). The IMO Convention for Safety of Life At Sea (SOLAS) Regulation V/19.2.4 requires all ships of 300 gross tonnages (GT) and upwards, cargo ships of 500 GT and upwards engaged on international voyages as well as passenger ships with all size should be fitted with AIS. Vessels fitted with AIS can be tracked by AIS base station [7].

AIS represents additional data source for radar. There are 27 message types that can be transmitted by AIS and each message has a specific purpose [8]. Compared to the radar system, AIS provides information about the vessel e.g. position, Maritime Mobile Service Identity (MMSI), vessel type, vessel dimension, Speed Over Ground (SOG), Course Over Ground (COG), navigational status, Rate of Turn (ROT), and heading. This information can be transformed into some useful information for maritime traffic analysis e.g. vessel path prediction and collision avoidance. AIS uses a transmission protocol called Self Organization Time Division Multiple Access (SOTDMA). This protocol allows AIS to be an autonomous active in the vessels.

AIS automatically broadcast ship information and voyage data at regular time interval. This information can be received by transceiver base station or VTS (Vessel Traffic Service). There are 2 types of AIS, Class A and Class B. Different type of ship carries a different class of AIS. Each AIS class transmits the data in a different time interval. Class A is designated for all vessels mentioned by SOLAS and Class B is optional. The regulation that requires AIS is to be fitted onboard the ship is effective for all ships by December 31st, 2004 (requirements by IMO).

AIS has some advantages over radar, e.g. less affected by weather condition, low power consumption, and lower operational cost than radar.

AIS operates on two VHF channels i.e. 162,975 MHz (for ship to ship) and $162.025 \mathrm{MHz}$ (for ship to the base station). AIS sends the following data every 2 to 10 seconds depending on the vessel speed while underway, and every 3 minutes while vessel is anchoring [8]. AIS data is encoded as a 6-bit text, similar to SMS (Short Message Service) in mobile phone. AIS data can be used by owner, government, and operator of marine infrastructures such as seabed cable or pipeline to monitor the activities of vessels operated close to their assets in real time. This information could avoid marine accidents between ships to offshore platform.

\subsection{AISITS}

AISITS is a system developed by ITS using AIS device to get vessels information. The system is majorly used to monitor the safety of offshore facilities such as subsea pipeline and offshore platform. The other features of AISITS is useful for helping the port authority to inspect ships which have the highest hazard score before entering the port. AISITS has equipped with an alert to warn the port authority. Currently, there are 3 main features of AISITS i.e. Pipeline Early Warning System (PEWS), Ship Inspection, and Ships Tracking or Ship Movement Recording.

AISITS obtains the data from many base stations. The positon of subsea pipeline or offshore platform then will be compared to the data and the comparison results are used by the system to analyze the danger score of the ships against the object (subsea pipeline or offshore platform). If the danger score is high, the system will show an alert. An alert will be sent automatically via 
visualization in LCD projector (on the computer), SMS, E-Mail, and mobile phone notification. AISITS has some advantages compared to other similar developed products such as:

- AISITS is the first AIS system in Indonesia for offshore facilities or marine installation.

- Low cost to build the system.

- AISITS is equipped with an early warning if there are vessels which has potential hazard to the object because the vessel's position close to the object.

- AISITS can integrate many base stations and control them in one control room.

- It determines the inspection score by using scientific solver. This feature can be used by the port authority to inspect all the vessels in the port more efficiently.

- An easiness monitoring because it can be remotely monitoring the port traffic anywhere such as office, home, campus or any places.

- Capability to predict the ship's heading using artificial intelligence.

- AISITS uses down sampling method to reduce a big data from AIS data stream.

\subsection{AISITS Structure}

AISITS has 4 structure types as shown in Figure 2 and every structure has a different function. This is the structure of AISITS:

1) AIS Decoder

Based on ITU (International Telecommunication Union) standard, AIS has 27 message types and each of them has different specifications and utilities. AISITS uses some messages from AIS data to develop the system. These messages are decoded by AISITS and stored in the database. The stored data can be viewed anytime on the website or mobile phone and can show the movement of vessels. There are several data that have been used to determine whether the ships are operated close to the marine facilities e.g. navigation status, heading/course of vessel, velocity of ship and last time ship to sends a data.

2) Remote AIS Integration

AISITS can control many AIS base stations in one control room. It can help port authorities to monitor ships from other locations at once. User can access it via website or mobile phone and can make a decision faster if there are accidents happening at the port.

3) AIS Data Stream

AISITS gets AIS data from many base stations and the data are send continuously like a data stream. The data stream needs a high capacity storage in the system. AISITS uses down sampling method to reduce the capacity required by the data AIS Historical. AISITS can assist forensic analysis to handle marine accident investigations such as collisions between ships, collision between ships and offshore platforms, and ships lost due to bad weather.

Based on the structures, AISITS is the first VTS (Vessel Traffic Service) in Indonesia and can monitor all ship traffics. The future development plan of AISITS will produce VTMIS (Vessel Traffic Management Information System). The goals of AISITS as VTMIS are:

- To help the port authority as a billing system for every ship which enters the port.

- Berth management system for all vessels.

- AISITS becomes a dispatch system.

- As Tugs and Pilots management system at the port. 


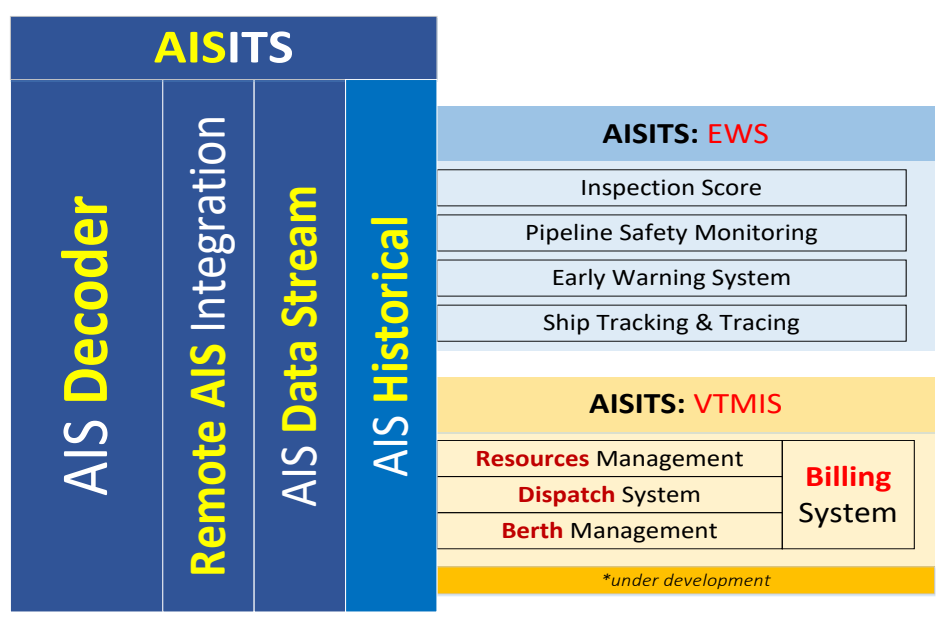

Fig. 2. AISITS structure

\section{Features of AISITS}

There are 3 main features of AISITS i.e. Pipeline Early Warning System (PEWS), Ship Inspection, and Ships Tracking or Ship Movement Recording [9].

\subsection{Pipeline Early Warning System (PEWS)}

Most of Indonesia oil and gas is at sea, so the offshore technology and conventional onshore technology is used in integrated manner to carry out exploration and exploitation. The technology used at least consist of a wellhead, subsea manifolds, subsea flow lines, offshore platforms, floating production units, export pipeline and an onshore receiving facility. There are some potential hazards that may occur, particularly for subsea pipelines and offshore platforms associated with the ship operation at sea, i.e.: drop anchor, anchor drag, sinking ship, vessel-platform collision, etc. [10].

Early Warning System (EWS) is built utilizing subsea pipeline and offshore structure position data and dynamic AIS data obtained from vessel in real time. The relative distance between vessel and subsea pipelines or offshore platform can be calculated accurately in real time. The boundaries of the restricted and exclusion area of subsea pipelines and offshore platform can also be used as EWS notification trigger. Overlay technique is also used to complement the EWS interface so that the movement of vessel and relative distance to the subsea pipelines and offshore platforms can be monitored visually. Visual monitoring is usually required by the port authorities and the owners of the platform. The display of AISITS Pipeline Early Warning System (PEWS) is presented in Figure 3.

When there is a vessel operated near to the pipeline and has a high risk against the pipeline, the warning will be triggered. It can prevent the vessel crash the pipeline. This feature is also used as a warning system to avoid the collisions between ships and offshore platform. According to the method of research about colouring scheme that has been conducted in 2011, the level of alert is represented by different colours i.e. red, yellow and green. The detail description of the colours as following:

- Red alert means the ship is in a location near to the pipeline and the navigational status of the ship is at anchor or moored.

- Yellow alert means the ship is also close to the pipeline, but the ship's navigational status is unknown (not at anchor or not moored).

- Green alert indicates the ship is located in the safe area and far to the pipeline. The alert with this colour can be ignored by port authority. 


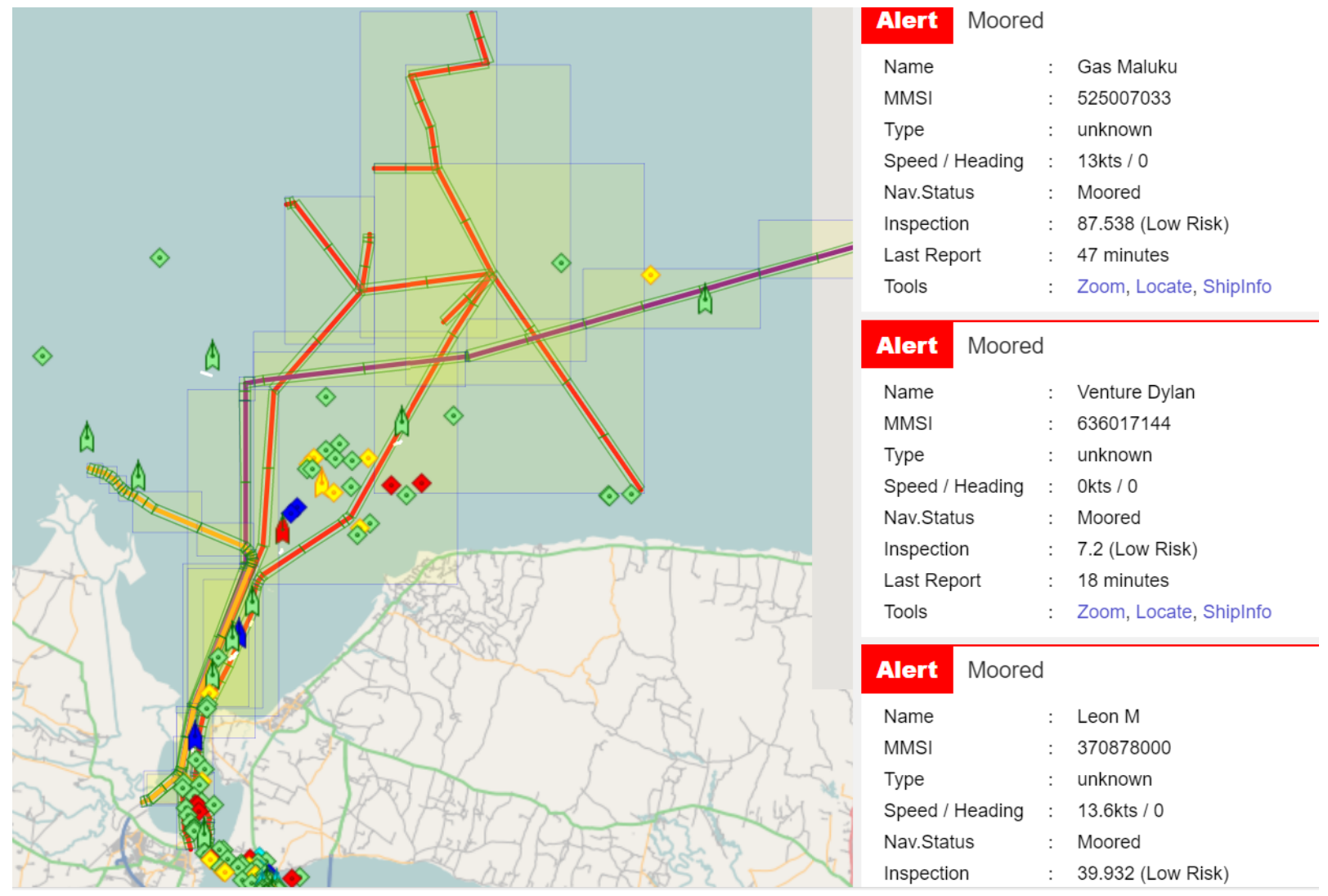

Figure 3: AISITS Pipeline Early Warning System (PEWS).

\subsection{Ship Inspection}

Ship inspection feature has been developed and conducted in 2012. The main purpose of this feature is to provide the ship inspection. This feature utilizes a scoring system to determine which ships have a high risk that must be inspected before or after entering the port. The criteria to calculate the inspection score are shop's age, ship's type, class of ship and ship's flag [5]. Figure 4 shows the inspection score of ships. There are 3 colours that represent the risk level:

- Red ship shows that the ship has a high risk to inspect by port authorities and/or syahbandar. (Score is more than 200)

- Yellow ship means that the ship has medium risk. The ship is optional to be inspected. (Score is between 100 and 200)

- Green ship indicates that the ship has low risk. The ship may not to be inspected. (Score is between 0 and 100). 


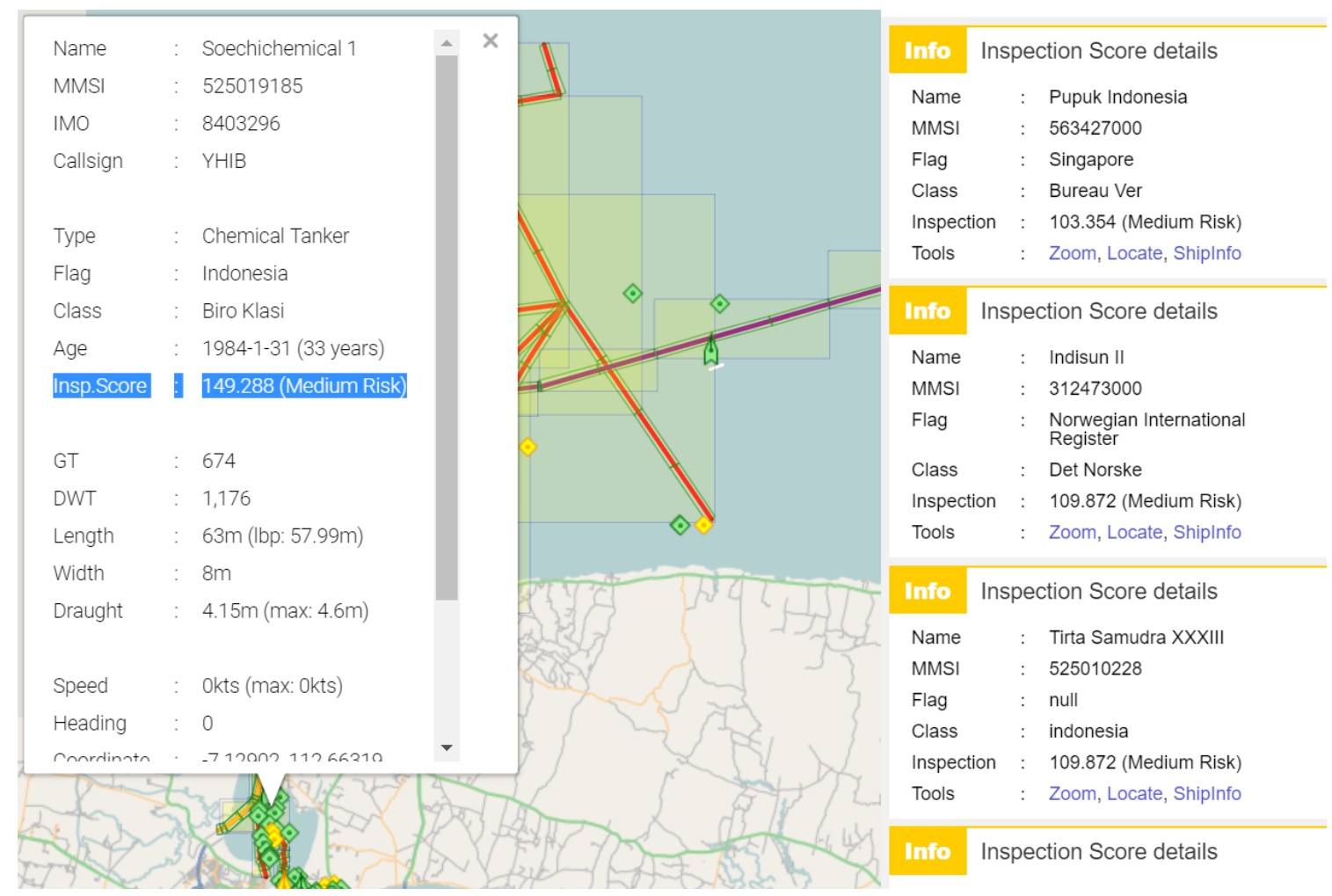

Figure 4: Inspection score.

\subsection{Ship Tracking}

Taking into consideration that AIS can transmit static and dynamic data regularly in seconds, AISITS utilizes AIS to real timely track the position of ships and mapped on the system. This feature enables ship owner, port authority, and others to know the exact position of a certain ship.

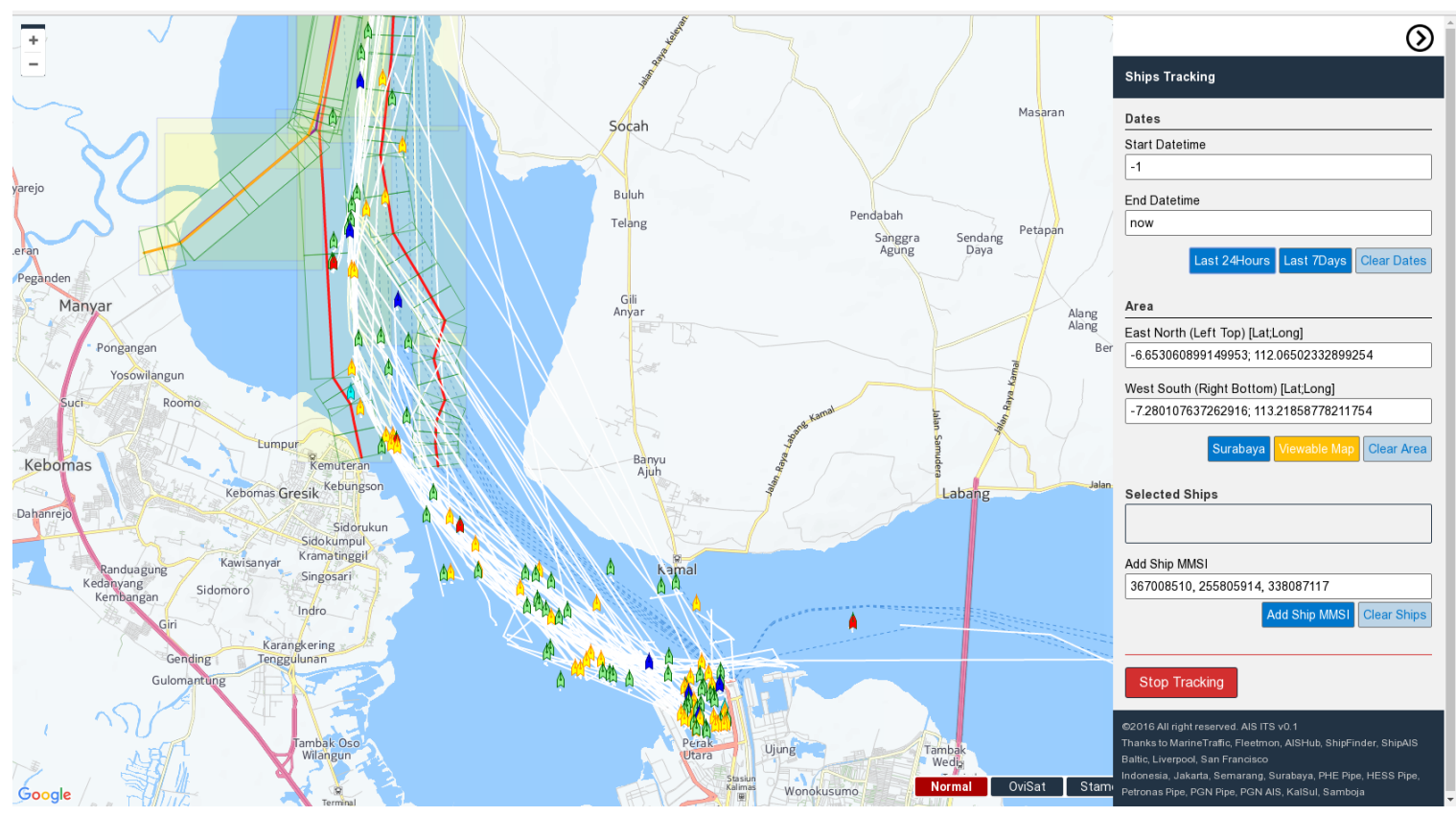

Figure 5: AISITS ship tracking. 
AISITS also provides a feature to reveal information on vessel's path way for a certain period, in a certain location. This provides a flexible way to perform a forensic analysis of ship collision/incident for further purposes. In the case of Indonesia, KNKT (national maritime safety board), insurance company, and other relevant parties can use the application functionally. Figure 5 shows the display interface of a vessel tracking system.

\section{Future Development of AISITS}

This paper shows some features of AISITS developed by the Laboratory of Reliability and Safety, Department of Marine Engineering. Currently, some further applications are under development, such as:

1) AISITS for port management. This feature will maximize the use of AIS data to real timely manage the pilotage of ships at port, berthing services, cargo loading/unloading, tug services and others. Making use of the feature, online single billing system could be initiated, and port company can provide an accountable and fair services to all vessels.

2) AISITS collision avoidance. The combination of AIS data and artificial intelligence (AI) could provide a precise prediction on how ship must response hazard condition on sea. AISITS ship tracking could be a backbone for such a development.

3) AISITS ship grounding avoidance. Overlaying digital bathymetry chart and AISITS map system could provide a functional alert system to avoid vessel to be grounded. The algorithm of under keel clearance (UKC) could be utilized as a basis in designing the alert system. When a vessel approaching an area where its UKC less than the safety requirement, then an alert system is initiated to enable ship's master response to the situation.

4) Integration of AISITS with CCTV and Radar will increase the functionality of the AISITS features. Real time monitoring system can be managed not only digitally, but also visually.

\section{Conclusion}

AIS based real time early warning system developed here undoubtedly provide instrument to increase the safety operation of ships and marine installation. The structure of AISITS explained in this paper also provides flexibility in accommodating other applications including integration with radar and CCTV.

Though so, an extensive testing is further required to ensure the applicability and reliability of AISITS. Extending the coverage of AISITS is another issue to cover wider maritime area.

\section{References}

[1] Indonesian Shipping Court, "Rekapitulasi data kecelakaan kapal,” Mahkamah Pelayaran Kementerian Perhubungan Indonesia, Jakarta, 2009.

[2] IMO, IMO Resolution MSC.74(69), Annex 3, Recommendation on performance standards for an universal shipboard automatic identification system(AIS). 2001.

[3] A. C. Bukhari, I. Tusseyeva, B.-G. lee, and Y.-G. Kim, "An intelligent real-time multi-vessel collision risk assessment system from VTS view point based on fuzzy inference system, ” Expert Syst. Appl., vol. 40, no. 4, pp. 1220-1230, 2013.

[4] K. B. Artana, D. DP, and T. P. Masroeri, "Combining AIS data and fuzzy clustering to measure danger score of ships," J. Marit. Res., vol. 1, no. 1, pp. 33-41, 2011.

[5] D. Masroeri AA, Artana KB, Pitana T, "A review on some research issues on AIS to improve the ship safety operation at sea," J. Marit. Res., vol. 2, no. 1, pp. 11-23, 2012.

[6] T. Pitana, E. Kobayashi, S. Koshimura, K. Onoda, and Rusmanto, "25 Evacuation Assessment of a Large Passenger Vessel due to Tsunami Attack,” J. Japan Soc. Nav. Archit. Ocean Eng., no. 8, pp. 205-217, 2008. 
[7] IMO, The IMO Convention for Safety of Life At Sea (SOLAS) Regulation V/19.2.4. 2002.

[8] ITU-R, M.1371 : Technical characteristics for an automatic identification system using time-division multiple access in the VHF maritime mobile band. 2014.

[9] AISITS, “AISITS Features." [Online]. Available: http://ais.its.ac.id/aisits-features/. [Accessed: 22-Jan-2018].

[10] K. B. Artana and A. A. B. Dinariyana, Teori Keandalan Sistem dan Aplikasinya. Surabaya. Guna Widya. Surabaya: Guna Widya, 2013. 